\title{
Fault analysis and maintenance of the hydraulic system of coal mining machinery
}

\author{
Qingsong $\mathrm{Gu}^{1, \text { a }}$ \\ ${ }^{1}$ Weihai vocational college, Shandong 264201, China \\ agqs65@163.com
}

\begin{abstract}
Keywords:Coal mining machinery; hydraulic system; fault
Abstract. With the continuous development of science and technology, mechanical work in the coal, the coal mining machinery hydraulic system has been more common, only the construction of a systematic dimension and control hierarchy management, in order to ensure the inspection results accord with the actual requirements of management. Technical staff in the establishment of fault analysis and control measures at the same time, it is necessary to conduct in-depth study and a comprehensive analysis of the corresponding research structure. In this paper, the coal mining machinery hydraulic system structure is briefly analyzed, and discussed the system fault and maintenance mechanism, in order to provide valuable references for related equipment maintenance and management personnel.

In recent years, the continued implementation of China's coal mining project development process, the use ratio of hydraulic equipment and the frequency of use is also increasing, how to construct the system of management mechanism and management dimension, the relevant departments need to systematically deal with the actual demand, and the hydraulic equipment maintenance intensive, so as to establish prevention and maintenance measures to ensure the production operation and management effect at the same time, further improve the overall effectiveness of the work.
\end{abstract}

\section{Hydraulic system of coal mining machinery}

The coal mining machinery hydraulic system is mainly composed of brake, clutch and gear shifting device, convert fluid pressure into mechanical energy, which is environmentally friendly and effective use value, and can use the actuator directly to the pressure into power. Therefore, in the process of carrying out the actual management process, if the hydraulic system failure, will cause the influence of transfer force of the gearbox to shift operation effectively, the effect of the corresponding processing equipment and the actual hydraulic structure will lose its effectiveness.

In the hydraulic system of coal mining, the most basic element is the power component, is the motive of the mechanical energy into the main path of liquid pressure pump hydraulic system with power on the construction of a systematic support system, to ensure the power effect in line with the actual needs. Secondly, is the control system of the structure, is mainly a series of hydraulic valve structure, the liquid flow rate and direction parameters such as systematic analysis and pressure adjustment, combined with different control mechanism of the hydraulic system control element stress management. It is worth mentioning that, in the control components, pressure control valves, flow control valves and directional control valve is a very important parameter structure. In addition, the coal mining hydraulic system, the implementation of components also has a very important role to ensure the effectiveness of the load rotation and reciprocating process. And by means of auxiliary components and hydraulic oil, to ensure the normal operation of the whole hydraulic control system.

\section{Coal mining machinery hydraulic system fault analysis}

The noise problem of hydraulic system in hydraulic system of coal mining machinery. In the coal mining machinery hydraulic system noise problems, it is necessary in order to carry out effective analysis and centralized inspection of the actual situation. If the actual oil viscosity, you need to select the appropriate add oil, if the internal surface of 
oil is low, to the actual resistance of the oil suction pipe and blockage of structure analysis, only to ensure the stability of oil liquid parameters, in order to improve the overall effect, ensure the flow in line with the actual, and to a detailed analysis of the effect and the degree of bending section runner structure. It is worth mentioning that the air into the pump equipment, it will lead to a series of adverse problems, and even make the mechanical system of vibration fault. Therefore, to conduct in-depth analysis of joint and screw structure, ensure the effectual treatment of air and cavitation structure, ensure effective sealing equipment and level structure inspection mechanism, and also provide convenience for the optimization of the exhaust effect.

Hydraulic system traction problem of hydraulic system of coal mining machinery. In the hydraulic system of coal mining machinery, the traction system is also a very important parameter structure. If the fault occurs, the whole system will be paralyzed. One of the reasons, first, the lack of coal mining machinery hydraulic system, the amount of oil, will lead to the emergence of a serious leakage of auxiliary pump, auxiliary pump in a timely manner to ensure the efficiency of its operation can be optimized. Second, the safety of the device to set the value of the law is low, it will cause the entire system failure, to be set [1]. Third, bad cooling or high temperature will lead to the number of numerical pressure and cooling water does not meet the standards, to ensure that quickly adjusted to the rated value. Fourth, the motor of coal mining machinery hydraulic system and main pump appeared serious leakage, will lead to the effectiveness of the main pump motor structure and the effect of the oil pressure value is low, will make the traction hydraulic system show a reduction problem, and even lead to oil leakage problems, the need for timely replacement of the sealing element and tighten sure, the optimized management dimension and management effect.

Hydraulic system pressure problem of hydraulic system of coal mining machinery. In the actual application process, the structure of the whole system if the instability problems will be combined with the actual needs of the construction of systematic management, promise between management model and management dimension can form a hierarchy of effective control. However, if there is plenty of dust and impurities on the throttle channel, it will lead to instability of the check results. Relevant technical personnel need to be on the relevant parameters of the relief valve and the setting value of the applicable inspection and control, and to ensure that the phase height and seal compliance standards. It is built on this, in order to better maintain the effective operation of the hydraulic system of coal mining machinery, it is necessary to carry out the overall management of its sealing.

Stability of hydraulic system in hydraulic system of coal mining machinery.In the application process of coal mining machinery hydraulic system, the hydraulic stability is also very important, technical personnel should be combined with the actual needs of the construction of the management mechanism of the system, the hydraulic stability problem of insufficient power to give the corresponding attention. Weak system overheating problems, you need to focus on checking the safety valve pressure regulating the value of focus, and the use of pressure regulation and air valve parameters such as the integrity of management. 
In addition, there are impurities in the safety valve, did not clean up in time, will lead to hydraulic stability affected. If the safety valve spring lost the basic operational capacity and overall control function, it will lead to the overall operating parameters affected by the scale, the need for technical support, the focus of its spring replacement. In the hydraulic system of coal mining machine will cause the safety valve operation is affected, and the need for specialized personnel according to the actual problems of setting check and fine adjustment to ensure the normal pressure of. It is worth mentioning that, in the management of personnel to concentrate on the repair of the pump in accordance with the orderly operation of the standardized installation, only to achieve the normalized management model [2].

Hydraulic system oil supply problem in hydraulic system of coal mining machinery. For the whole system, it is necessary in order to analyze the problems of the hydraulic system which is difficult to supply oil to ensure the overall management effect and the centralized management. In the process of practical design and application, it is necessary to deal with the faulty location of the structure and the related parameter model. In order to solve the problem of fault, we should find the fault location effectively, and guarantee the comprehensive control of the maintenance project. For correct one way pump steering should also give the necessary attention, it will lead to the coal mining machinery hydraulic system is difficult to supply problems. The need for technical and equipment maintenance personnel to carry out the computation. The oil viscosity is too high, also need technical structure of the tank, first of all, make sure the tank, and replaced with low viscosity oil, secondly, equipment failure may also be caused by the blockage problems related to oil pipeline, it should be conducted on the oil filter and oil pipeline

Overall inspection and systematic arrangement, the exclusion of the blockage of the pipeline, so as to ensure the optimization of the operation dimension and operational effect. If the equipment in the oil temperature is too low, there will be leakage problems, technical personnel should be specific to the fuel tank to check the leak location and take effective repair mechanism, in accordance with the requirements and the relevant standards in line with the actual gas.

Leakage of hydraulic system in hydraulic system of coal mining machinery. In the light of the actual situation, the system should be utilized to judge the leakage of the oil tank. For the hydraulic system of coal mining machinery, the leakage of the hydraulic system is more prominent, mainly in the oil leakage.

\section{Coal mining machinery hydraulic system fault maintenance mechanism analysis}

In the management and maintenance system, in the process of the establishment, relevant technical management personnel to ensure the effectiveness of management mechanism and management measures, and earnestly safeguard the orderly control model, and to ensure the matching degree between management information and management requirements.

The prevention of coal mining machinery hydraulic system maintenance". In the process of carrying out the actual management of the project, to actively implement the requirements of management mechanism and management, and earnestly safeguard the 
effectiveness of the management model and management standards, to ensure that only the management failures can be effective prevention and management, in order to reduce the occurrence probability of [3] fundamentally. Therefore, "prevention first" as the management benchmark has a certain practical significance. In order to make sure the optimization of the control effect, the technical staff should combine with the actual needs and focus on the corresponding fault. It is worth mentioning that, in the fault before, must have the corresponding sign, is based on this, the technical staff to integrate technology on the structure and failure problems, so as to ensure the integrity of the fault, in order to further optimize the management process to provide direct information, ensure based on careful management, the cause of the fault comprehensive analysis and fault movement. Only to ensure effective prevention work, to carry out systematic work, needs technical personnel to carry out centralized control and comprehensive prevention and control, in order to reduce the probability of fault occurrence, to ensure the complete operation of the coal mining machinery hydraulic system.

The conventional management of hydraulic system maintenance mechanism of coal mining machinery. In view of the coal mining machinery hydraulic system maintenance measures and operational status analysis, by the fault of the careful search and centralized control at the same time, actively implement the fault collection and control mode. Technical management personnel should according to the actual problems of the construction management model and basic management dimension, and the hydraulic system of the abnormal sound that, based on the timely registration, to ensure the optimization of management, but also to the effectiveness of its operation dimensions, ensure to determine the location of the sound is not always guaranteed, between the management structure and management level model maintenance effect, working principle and technical personnel to carefully check the local parts and project structure [4]. Targeted to select the appropriate parameters, the combination of analysis and thinking, centralized management and control of the causes of failure and integrated control model, to ensure that the position can be deduced. Based on this, technical personnel based on the corresponding control measures, through parts of the surface of the touch, effective to understand and use the parameters such as wear parts, not only to ensure that the temperature of the structure model to fit the actual situation, detection method of application of the system is advanced and effective test method, preliminary diagnosis after the empirical analysis and comprehensive control card.

Application of hydraulic oil in hydraulic system maintenance mechanism of coal mining machinery. In the hydraulic system, the transmission of the signal and the transmission of hydraulic oil power structure, the need for relevant management personnel with the actual needs and control measures for comprehensive analysis and centralized treatment. The antirust and lubrication effect of the use of hydraulic oil, in the actual application process, the hydraulic oil system failure is mainly due to oil pollution, which mainly include the gelatinous material, mixed with air and impurities, and also relates to water, will lead to the overall operation of equipment structure and the actual effect is seriously affected, centralized treatment and comprehensive control related technical management personnel according to the actual situation, especially for the oil 
pollution and the temperature rise of the systematic analysis on stability management of valve operation, and ensure the effectiveness of maintenance management system and operation structure. In the process of fault analysis, not only to the hydraulic system of the working principle of a comprehensive analysis, but also in order to check the fault location after the comprehensive consideration, to achieve the balance of economic and political benefits. To carry out systematic maintenance of the inspection site, in addition to maintenance, but also to establish a dynamic maintenance mechanism and control model to further improve performance stability.

\section{Conclusion}

In a word, in the actual process of project management in the establishment, relevant technical staff to carry out systematic control and treatment according to the actual fault, not only to the fault location on the amendment to the causes of failure of detailed analysis, in ensuring the development prospects of the enterprise model fit at the same time, a comprehensive consideration of economic benefit and social benefit. Also to ensure the management effect, to a certain extent, for the sustainable development of coal mine laid a solid foundation.

\section{References}

[1] Zhao Z, Zhang J, Wang S, et al, Fault Prediction and Maintenance of Non - Periodic Incipient Fault for Hydraulic Tube Tester. Asian Journal of Control, 16(6),pp:1670-1678,2014.

[2]Ruhi S, Karim M R,Selecting statistical model and optimum maintenance policy: a case study of hydraulic pump. SpringerPlus, 5(1),pp:969,2016.

[3]Han M, Zhao W, Cheng Y, et al. Concept Design of Water Hydraulic Circuit for Manipulator of CFETR Blanket Maintenance. Journal of Fusion Energy, 34(4),pp:765-768, 2015.

[4]Gamauf M, Hydraulic system maintenance--tips and advice : though safe and reliable, vigilance is essential during a repair. Econometrica, 59(3),pp:711-730,2014. 